\title{
Características das emissões otoacústicas em lactentes expostos à medicação ototóxica
}

\section{Characteristics of otoacoustic emissions in infants exposed to ototoxic drugs}

\author{
Andreza Ferreira dos Santos ${ }^{1}$, Alessandra Spada Durante ${ }^{2}$, Katia de Almeida ${ }^{3}$, Carlos Kazuo Taguchi ${ }^{4}$, \\ Maria Cecilia Greco ${ }^{5}$
}

\begin{abstract}
RESUMO
Objetivo: Analisar as emissões otoacústicas (EOA) em lactentes submetidos a tratamento por medicação ototóxica no período neonatal. Métodos: Foi realizada a captação das EOA por transiente (EOAT) e das EOA produto de distorção (EOAPD) em 40 lactentes: 14 lactentes submetidos a drogas ototóxicas por, no mínimo, cinco dias (grupo estudo) e 26 lactentes sem risco auditivo e sem intercorrências (grupo controle). A análise estatística do conjunto de dados foi efetuada utilizando-se os testes não-paramétricos de Wilcoxon e Mann-Whitney, e a técnica de intervalo de confiança para média. Resultados: Foram observados menores níveis de resposta e relação sinal/ruído nas EOAPD para o grupo estudo, com valores estatisticamente significantes para as frequências de 3000, 6000 e $8000 \mathrm{~Hz}$. Conclusão: A ação dos ototóxicos pode ser observada pela resposta típica das EOA em altas frequências, o que foi mais bem avaliado pelas EOAPD.
\end{abstract}

Descritores: Lactente; Fatores de risco; Emissões otoacústicas espontâneas/efeitos de drogas

\section{INTRODUÇÃO}

O uso de medicamentos ototóxicos pode acarretar uma deficiência auditiva adquirida, em que há uma perda parcial ou total da função coclear em decorrência do uso de drogas, geralmente com finalidades terapêuticas ou por exposição a substâncias tóxicas. O grau de severidade depende da predisposição individual, dose, duração do tratamento, via de administração, idade, tendência familiar e/ou de eventual dano prévio à orelha interna ${ }^{(1)}$.

Trabalho desenvolvido no Curso de Fonoaudiologia da Faculdade de Ciências Médicas da Santa Casa de São Paulo - FCMSCSP - São Paulo (SP), Brasil. (1) Especialista em Audiologia Clínica; Fonoaudióloga clínica - São Paulo (SP), Brasil.

(2) Doutora, Professora Assistente do Curso de Graduação em Fonoaudiologia da Faculdade de Ciências Médicas da Santa Casa de São Paulo - FCMSCSP - São Paulo (SP), Brasil.

(3) Doutora, Professora Adjunto da Faculdade de Ciências Médicas da Santa Casa de São Paulo - FCMSCSP - São Paulo (SP), Brasil.

(4) Doutor, Professor Adjunto da Faculdade de Ciências Médicas da Santa Casa de São Paulo - FCMSCSP - São Paulo (SP), Brasil; Professor Adjunto da Universidade Federal de Sergipe - UFS - Aracaju (SE), Brasil.

(5) Professora instrutora do Curso de Graduação em Fonoaudiologia da Faculdade de Ciências Médicas da Santa Casa de São Paulo - FCMSCSP - São Paulo (SP), Brasil.

Endereço para correspondência: Andreza Ferreira dos Santos. Estrada Samuel Aizemberg, 254, Jd. Santa Maria, São Bernardo do Campo (SP), Brasil, CEP: 09851-550. E-mail: andrezade@itelefonica.com.br

Recebido em: 26/2/2009; Aceito em: 15/6/2009
Estes fármacos, principalmente a amicacina e a gentamicina são amplamente utilizados no tratamento de infecções em recém-nascidos, com objetivo terapêutico e profilático, proporcionando um decréscimo na mortalidade perinatal, resultante do incremento nos cuidados neonatais. Além disso, aumentam a sobrevida de crianças nascidas com baixo peso e muito baixo peso, apesar de haver a possibilidade de sequelas, entre as quais a deficiência auditiva ${ }^{(2)}$. Essas drogas lesam as estruturas sensoriais da orelha interna, podendo afetar tanto o sistema auditivo quanto o vestibular. Vários fatores podem influenciar no potencial da toxicidade, como: o agente, a dose, a duração, a função renal, o uso prévio de outras drogas e a susceptibilidade individual ${ }^{(3)}$.

A origem dos aminoglícosideos iniciou-se com o desenvolvimento da estreptomicina e foi produto de uma pesquisa científica com substâncias antibacterianas. Em 1960, foi isolada a gentamicina, que é o mais utilizado dos aminoglícosideos e é produzido por espécies de Actinomyces micronospora. A amicacina (primeiro aminoglícosideo semi-sintético, 1972), assim como outros aminoglicosídeos, foram desenvolvidas para suplantar a resistência da Pseudômona ${ }^{(4)}$.

Tratando-se de drogas potencialmente ototóxicas, capazes de lesar de forma irreversível as células da orelha interna, é importante a monitorização dos pacientes que serão submetidos a tratamento com aminoglicosídeos. Um dos testes mais utilizados na bateria audiológica para o diagnóstico precoce e acompanhamento audiológico de crianças pequenas são as 
emissões otoacústicas (EOA), observando-se principalmente as respostas para as altas freqüências ${ }^{(5)}$.

As emissões otoacústicas evocadas são registradas após a apresentação de um estímulo acústico e estão presentes, em 99,9\% da população ouvinte normal; daí o seu valor clínico. As EOA são classificadas de acordo com o estímulo acústico empregado e as mais utilizadas são as emissões otoacústicas por estímulo transiente (EOAT) e as emissões otoacústicas por produto de distorção (EOAPD).

As EOAT são provocadas por um sinal transiente ou clique. Uma sonda é utilizada para apresentar o sinal-clique e para registrar a resposta. Uma série de estímulos-cliques é apresentada, em geral, em um nível de intensidade entre 80 e $85 \mathrm{~dB}$ NPS pico equivalente. As respostas são detectadas por um microfone dentro da sonda e, em geral, em uma janela de $20 \mathrm{~ms}$.

As EOAPD ocorrem como resultado de um processo não linear da cóclea. Quando dois tons são apresentados à cóclea, a distorção ocorre na forma de outros tons que não estavam presentes nos dois tons dos sinais estimuladores. Estas distorções são combinações de tons ou harmônicos, que estão relacionados aos tons estimuladores de uma forma matemática previsível. Os dois tons usados para provocar as EOAPD são, por convenção, denominados f1 e f2 apresentados em uma relação $\mathrm{f}_{1} / \mathrm{f}_{2}=1,2$ e com intensidades $65 \mathrm{~dB}$ e $55 \mathrm{~dB}$, respectivamente. O produto de distorção mais intenso ocorre na frequência representada pela equação $2 \mathrm{f}_{1}-\mathrm{f}_{2}$. Pares de tons puros são apresentados ao longo do campo de frequência de 1000 a $8000 \mathrm{~Hz}$.

No registro das EOA, são considerados vários parâmetros para a sua interpretação. Os mais importantes são a magnitude da resposta e a reprodutibilidade média. A magnitude de resposta é a intensidade da emissão originada na cóclea, em função do ruído expresso em decibels nível de pressão sonora (dB NPS), e é tanto maior quanto maior for a atividade biomecânica coclear ${ }^{(6)}$.

Em função das alterações que as drogas ototóxicas podem provocar na audição, elas são consideradas um indicador de risco para a deficiência auditiva ${ }^{(7)}$. A hipótese deste estudo é de que, em função da ototoxicidade, os lactentes expostos a estas drogas no período neonatal, apresentariam uma função coclear reduzida e, consequentemente, menor magnitude de resposta na captação das EOA. A caracterização das EOA nestes lactentes possibilitaria, além da detecção precoce da disfunção auditiva, o estabelecimento da intervenção adequada, propiciando o máximo desenvolvimento de comunicação destas crianças.

Assim, o objetivo deste estudo é caracterizar as emissões otoacústicas em lactentes submetidos a tratamento por medicação ototóxica.

\section{MÉTODOS}

\section{Sujeitos}

Foi realizado um estudo de caráter exploratório, prospectivo e transversal, no qual foram avaliados 40 lactentes, nascidos na Maternidade da Irmandade da Santa Casa de Misericórdia de São Paulo, no período entre fevereiro e junho de 2007. Todos esses lactentes foram encaminhados à Clínica de Fonoaudiologia, onde foram submetidos ao exame de captação das emissões otoacústicas. Os lactentes foram separados em dois grupos: grupo comparação e grupo estudo.

\section{Grupo comparação}

O grupo comparação foi composto de 26 lactentes, dos quais 13 eram do gênero feminino e 13 lactentes do gênero masculino. Todos nascidos a termo, sem intercorrências pré e perinatais e sem indicadores de risco para alteração auditiva e que apresentaram respostas dentro dos parâmetros de normalidade nas EOA em ambas as orelhas.

\section{Grupo estudo}

O grupo estudo foi composto por 14 lactentes, cinco do gênero feminino e nove do gênero masculino, expostos a medicação ototóxica por, no mínimo, cinco dias, nascidos a termo ou com idade gestacional corrigida entre 40 e 41 semanas, na data da avaliação.

\section{Equipamento}

As medidas de EOA foram realizadas por meio do analisador de emissões cocleares Capella-Madsen (GN Otometrics®), acoplado a um computador DELL® com monitor colorido de 17 polegadas e Processador Celeron, operando com o Windows Microsoft $₫$ junto à plataforma do software NOAH. Para captar as emissões foi utilizada uma sonda, conectada ao canal da unidade externa do equipamento.

Para a captação das EOAT foram empregados cliques de banda larga no modo não linear, eliminando a ocorrência de artefatos e viabilizando a aplicação clínica do experimento. A intensidade do estímulo variou de 75 a 80 dB NPS pico equivalente com duração de $40 \mu$ s e as respostas foram analisadas em janela de 3 a 12,5 ms, nas frequências de 1000, 1500, 2000, 3000 e $4000 \mathrm{~Hz}$.

Para a captação das EOAPD a medida de cada ponto de frequência envolveu a apresentação de dois tons puros primários, $\mathrm{f}_{1}$ e $\mathrm{f}_{2}$. Para esta pesquisa, seguiu-se o padrão sugerido pelo fabricante do equipamento, ou seja, relação das frequências $\mathrm{f}_{2} /$ $\mathrm{f}_{1}$ igual a 1,2 e intensidade de estímulos, respectivamente, de 55 a $65 \mathrm{~dB}$ NPS. As EOAPD foram pesquisadas nas frequências $\mathrm{f}_{2}: 1500,2000,3000,4000,6000$ e $8000 \mathrm{~Hz}$.

\section{Procedimento}

Este estudo foi submetido às normas do Comitê de Ética em Pesquisa da Faculdade de Ciências Médicas da Santa Casa de São Paulo (Protocolo de pesquisa n ${ }^{\circ}$ 195/6, aprovado em 30 de agosto de 2006).

Inicialmente, os pais dos lactentes foram informados sobre os objetivos da pesquisa e, estando em comum acordo com a pesquisa, assinaram um Termo de Consentimento Livre e Esclarecido.

Em seguida foram realizados os exames de EOAT e EOA- 
PD, em sala silenciosa e tratada acusticamente. Para a captação das EOA foi realizada a adaptação da sonda na primeira orelha a ser testada. A realização da avaliação se deu no período neonatal, sendo que no caso de lactentes nascidos prematuros, a idade gestacional, corrigida na data da avaliação, estava entre 37 e 41 semanas.

A análise estatística do conjunto de dados foi efetuada utilizando os testes não paramétricos de Wilcoxon e MannWhitney. Na complementação da análise descritiva, foi utilizada a técnica de Intervalo de Confiança para média. Em todos os testes, foi destacado o nível descritivo, sendo utilizado o nível de significância de 0,05 (ou 5\%) para rejeição da hipótese de nulidade.

\section{RESULTADOS}

Os medicamentos ototóxicos utilizados no grupo estudo foram amicacina, gentamicina e vancomicina, sendo que três lactentes foram tratados com vancomicina e 11 lactentes foram tratados com gentamicina. Dos lactentes tratados inicialmente com gentamicina, seis foram submetidos ao uso de amicacina e três submetidos, também, à vancomicina.

Para o grupo controle tanto o nível de resposta quanto a relação sinal ruído (S/R) das EOAT geral e por banda de frequências segundo orelha e gênero, foram maiores na OD para o gênero feminino do que na $\mathrm{OE}$, porém sem significância estatística (Tabela 1).

No grupo estudo, os níveis de resposta das EOAT geral e por banda de frequências segundo a orelha, a OE apresentaram resultados maiores do que a OD para o nível de resposta geral ( $\mathrm{p}=0,009)$. Pode-se observar, também, que para o nível de resposta quanto à relação $\mathrm{S} / \mathrm{R}$ das EOAT geral e por banda de frequências segundo gênero, apresentaram valores maiores para o gênero feminino, com significância estatística $(p=0,034)$ apenas para relação $\mathrm{S} / \mathrm{R}$ da resposta geral, assim como pode ser observado na Tabela 2.

Foi observada diferença entre as EOAT do grupo estudo e controle apenas na relação $S / R$ da banda de freqüência de $4000 \mathrm{~Hz}$ (Tabela 3).

Não foi observada diferença nos níveis de resposta e relação S/R das EOAPD por frequência entre os gêneros e entre as orelhas nas respostas das EOAPD de ambos os grupos.

As EOAPD apresentaram maiores níveis de resposta e relação $\mathrm{S} / \mathrm{R}$ para o grupo controle, com valores estatisticamente significantes para as frequências de 3000, 6000 e $8000 \mathrm{~Hz}$. Tal situação pode ser visualizada na Tabela 4 .

Tabela 1. Nível de resposta e relação S/R das EOAT geral e por banda de frequência, para o grupo controle, segundo orelha e gênero

\begin{tabular}{|c|c|c|c|c|c|c|c|c|c|}
\hline EOAT & Frequência & Orelha & Média & IC & Valor de $p$ & Gênero & Média & IC & Valor de $p$ \\
\hline \multirow{12}{*}{$\begin{array}{l}\text { Nível de resposta } \\
\text { dB NPS }\end{array}$} & \multirow{2}{*}{$1 \mathrm{kHz}$} & OD & 14,80 & 1,56 & \multirow{2}{*}{$0,041^{*}$} & Fem & 15,58 & 1,72 & \multirow{2}{*}{$0,003^{*}$} \\
\hline & & OE & 12,02 & 2,18 & & Masc & 11,03 & 1,80 & \\
\hline & \multirow{2}{*}{$1,5 \mathrm{kHz}$} & OD & 16,23 & 2,37 & \multirow{2}{*}{0,983} & Fem & 17,40 & 1,78 & \multirow{2}{*}{$0,070 \#$} \\
\hline & & OE & 15,45 & 2,02 & & Masc & 14,14 & 2,51 & \\
\hline & \multirow{2}{*}{$2 \mathrm{kHz}$} & OD & 19,23 & 1,87 & \multirow{2}{*}{0,240} & Fem & 19,01 & 1,80 & \multirow{2}{*}{0,452} \\
\hline & & OE & 17,46 & 1,59 & & Masc & 17,62 & 1,65 & \\
\hline & \multirow{2}{*}{$3 \mathrm{kHz}$} & OD & 22,33 & 2,30 & \multirow{2}{*}{0,983} & Fem & 22,55 & 2,15 & \multirow{2}{*}{0,411} \\
\hline & & OE & 21,26 & 1,73 & & Masc & 20,97 & 1,79 & \\
\hline & \multirow{2}{*}{$4 \mathrm{kHz}$} & OD & 18,91 & 2,41 & \multirow{2}{*}{0,777} & Fem & 19,89 & 2,25 & \multirow{2}{*}{0,280} \\
\hline & & OE & 18,68 & 2,21 & & Masc & 17,60 & 2,28 & \\
\hline & \multirow{2}{*}{ Geral } & OD & 27,45 & 1,79 & \multirow{2}{*}{0,408} & Fem & 27,81 & 1,69 & \multirow{2}{*}{0,244} \\
\hline & & OE & 26,44 & 1,37 & & Masc & 25,99 & 1,34 & \\
\hline \multirow{12}{*}{$\begin{array}{l}\text { Relação S/R } \\
\text { dB NPS }\end{array}$} & \multirow{2}{*}{$1 \mathrm{kHz}$} & OD & 9,08 & 3,37 & \multirow{2}{*}{$0,039^{*}$} & Fem & 7,61 & 3,18 & \multirow{2}{*}{0,934} \\
\hline & & OE & 4,55 & 2,08 & & Masc & 5,95 & 2,46 & \\
\hline & \multirow{2}{*}{$1,5 \mathrm{kHz}$} & OD & 16,07 & 3,21 & \multirow{2}{*}{0,396} & Fem & 17,35 & 3,19 & \multirow{2}{*}{0,431} \\
\hline & & OE & 16,10 & 2,99 & & Masc & 14,70 & 2,82 & \\
\hline & \multirow{2}{*}{$2 \mathrm{kHz}$} & OD & 19,91 & 2,50 & \multirow{2}{*}{0,469} & Fem & 18,16 & 2,09 & \multirow{2}{*}{0,196} \\
\hline & & OE & 18,14 & 18,95 & & Masc & 19,98 & 2,63 & \\
\hline & \multirow{2}{*}{$3 \mathrm{kHz}$} & OD & 20,57 & 19,55 & \multirow{2}{*}{0,794} & Fem & 20,17 & 2,34 & \multirow{2}{*}{0,630} \\
\hline & & OE & 19,05 & 20,85 & & Masc & 19,42 & 2,04 & \\
\hline & $4 \mathrm{kHz}$ & OD & 16,83 & 15,65 & 0.913 & Fem & 16,90 & 2,21 & 0385 \\
\hline & $4 \mathrm{NIIL}$ & OE & 15,75 & 15,90 & טוש, & Masc & 15,62 & 2,61 & U, \\
\hline & & OD & 15,16 & 15,60 & 0 & Fem & 14,25 & 1,92 & 0661 \\
\hline & Geral & OE & 14,05 & 13,75 & 0,171 & Masc & 14,99 & 2,17 & 0,664 \\
\hline
\end{tabular}

Legenda: $\mathrm{EOAT}=$ emissões otoacústicas transientes; $\mathrm{kHz}=$ kilohertz; $\mathrm{S} / \mathrm{R}=$ sinal/ruído; $\mathrm{dB}$ NPS = decibel nível de pressão sonora; OD = orelha direita; OE = orelha esquerda; IC = intervalo de confiança; Fem = feminino; Masc = masculino

* Valores estatisticamente significantes; \# Valores com tendência à significância estatística 
Tabela 2. Nível de resposta e relação S/R das EOAT geral e por banda de frequência, para o grupo estudo, segundo a orelha e o gênero

\begin{tabular}{|c|c|c|c|c|c|c|c|c|c|}
\hline EOAT & Frequência & Orelha & Média & IC & Valor de $p$ & Gênero & Média & IC & Valor de $p$ \\
\hline \multirow{10}{*}{$\begin{array}{l}\text { Nível de resposta } \\
\text { dB NPS }\end{array}$} & $1 \mathrm{kHz}$ & $\begin{array}{l}\text { OD } \\
\text { OE }\end{array}$ & $\begin{array}{l}16,84 \\
14,34\end{array}$ & $\begin{array}{l}8,37 \\
4,87\end{array}$ & 0,721 & $\begin{array}{l}\text { Fem } \\
\text { Masc }\end{array}$ & $\begin{array}{l}15,79 \\
15,46\end{array}$ & $\begin{array}{c}12,49 \\
3,23\end{array}$ & 0,165 \\
\hline & \multirow[t]{2}{*}{$1,5 \mathrm{kHz}$} & OD & 14,74 & 2,61 & \multirow{2}{*}{0,110} & Fem & 16,88 & 3,97 & \multirow{2}{*}{0,700} \\
\hline & & $\mathrm{OE}$ & 17,44 & 4,08 & & Masc & 15,57 & 3,18 & \\
\hline & $2 \mathrm{kHz}$ & $\begin{array}{l}\text { OD } \\
\text { OE }\end{array}$ & $\begin{array}{l}18,56 \\
18,17\end{array}$ & $\begin{array}{l}2,75 \\
4,25\end{array}$ & 0,415 & $\begin{array}{l}\text { Fem } \\
\text { Masc }\end{array}$ & $\begin{array}{l}19,65 \\
17,51\end{array}$ & $\begin{array}{l}3,46 \\
3,43\end{array}$ & 0,334 \\
\hline & \multirow[t]{2}{*}{$3 \mathrm{kHz}$} & OD & 21,66 & 4,72 & \multirow[t]{2}{*}{0,678} & Fem & 22,76 & 4,09 & \multirow[t]{2}{*}{0,700} \\
\hline & & OE & 20,80 & 5,34 & & & 20,21 & 5,17 & \\
\hline & \multirow{2}{*}{$4 \mathrm{kHz}$} & OD & 21,21 & 4,94 & \multirow{2}{*}{0,799} & Fem & 24,00 & 4,63 & \multirow{2}{*}{0,143} \\
\hline & & OE & 19,62 & 5,24 & & Masc & 18,03 & 4,66 & \\
\hline & \multirow{2}{*}{ Geral } & OD & 25,64 & 3,43 & \multirow{2}{*}{$0,009^{*}$} & Fem & 29,09 & 3,60 & \multirow{2}{*}{0,280} \\
\hline & & OE & 28,75 & 2,48 & & Masc & 25,93 & 2,60 & \\
\hline \multirow{12}{*}{$\begin{array}{l}\text { Relação S/R } \\
\text { dB NPS }\end{array}$} & \multirow{2}{*}{$1 \mathrm{kHz}$} & OD & 2,42 & 1,46 & \multirow{2}{*}{$0,05^{*}$} & Fem & 4,16 & 1,91 & \multirow{2}{*}{0,462} \\
\hline & & OE & 5,31 & 2,80 & & Masc & 3,67 & 2,54 & \\
\hline & \multirow{2}{*}{$1,5 \mathrm{kHz}$} & OD & 12,17 & 3,88 & \multirow{2}{*}{0,241} & Fem & 18,34 & 6,16 & \multirow{2}{*}{0,123} \\
\hline & & OE & 15,98 & 7,08 & & Masc & 11,23 & 4,84 & \\
\hline & \multirow{2}{*}{$2 \mathrm{kHz}$} & OD & 18,05 & 3,38 & \multirow{2}{*}{0,721} & Fem & 21,33 & 4,48 & \multirow{2}{*}{0,217} \\
\hline & & OE & 19,72 & 6,50 & & Masc & 17,26 & 5,12 & \\
\hline & \multirow{2}{*}{$3 \mathrm{kHz}$} & OD & 21,49 & 4,44 & \multirow{2}{*}{0,575} & Fem & 23,40 & 4,98 & \multirow{2}{*}{0,487} \\
\hline & & $\mathrm{OE}$ & 22,96 & 5,86 & & Masc & 21,44 & 5,11 & \\
\hline & \multirow{2}{*}{$4 \mathrm{kHz}$} & OD & 22,21 & 3,81 & 0646 & Fem & 23,86 & 4,86 & 0396 \\
\hline & & OE & 21,11 & 5,89 & 0,646 & Masc & 20,19 & 4,67 & 0,396 \\
\hline & 0 & OD & 13,80 & 3,10 & 0280 & Fem & 18,93 & 4,32 & \\
\hline & Geral & OE & 16,20 & 5,59 & 0,386 & Masc & 12,38 & 3,85 & $0,034^{\wedge}$ \\
\hline
\end{tabular}

Legenda: EOAT = emissões otoacústicas transientes; $\mathrm{kHz}=$ kilohertz; $\mathrm{S} / \mathrm{R}=$ sinal/ruído; $\mathrm{dB} \mathrm{NPS}=$ decibel nível de pressão sonora; OD = orelha direita; OE = orelha esquerda; IC = intervalo de confiança; Fem = feminino; Masc = masculino

*Valores estatisticamente significantes

Tabela 3. Nível de resposta e relação S/R das EOAT por grupo e banda de frequência

\begin{tabular}{|c|c|c|c|c|c|c|c|c|c|c|}
\hline EOAT & Frequência & Grupo & Média & Mediana & Desvio padrão & Q1 & Q3 & $\mathrm{N}$ & IC & Valor de $p$ \\
\hline \multirow{12}{*}{$\begin{array}{l}\text { Nível de resposta } \\
\text { dB NPS }\end{array}$} & \multirow{2}{*}{$1 \mathrm{kHz}$} & Controle & 13,41 & 13,00 & 5,08 & 10,55 & 17,45 & 44 & 1,50 & \multirow{2}{*}{0,965} \\
\hline & & Estudo & 15,59 & 13,50 & 11,97 & 9,88 & 17,93 & 20 & 5,25 & \\
\hline & \multirow{2}{*}{$1,5 \mathrm{kHz}$} & Controle & 15,84 & 16,70 & 5,67 & 14,00 & 18,80 & 44 & 1,68 & \multirow{2}{*}{0,873} \\
\hline & & Estudo & 16,09 & 16,05 & 5,55 & 11,60 & 18,53 & 20 & 2,43 & \\
\hline & \multirow{2}{*}{$2 \mathrm{kHz}$} & Controle & 18,35 & 18,75 & 4,55 & 15,43 & 20,53 & 44 & 1,35 & \multirow{2}{*}{0,862} \\
\hline & & Estudo & 18,37 & 18,30 & 5,63 & 14,20 & 21,85 & 20 & 2,47 & \\
\hline & \multirow{2}{*}{$3 \mathrm{kHz}$} & Controle & 21,80 & 21,00 & 5,26 & 18,43 & 24,95 & 44 & 1,55 & \multirow{2}{*}{0,942} \\
\hline & & Estudo & 21,23 & 21,25 & 7,93 & 18,80 & 25,08 & 20 & 3,47 & \\
\hline & \multirow{2}{*}{$4 \mathrm{kHz}$} & Controle & 18,80 & 18,20 & 5,94 & 14,93 & 22,80 & 44 & 1,76 & \multirow{2}{*}{0,268} \\
\hline & & Estudo & 20,42 & 20,75 & 8,04 & 17,23 & 23,85 & 20 & 3,52 & \\
\hline & \multirow{2}{*}{ Geral } & Controle & 26,94 & 26,70 & 4,13 & 23,83 & 29,28 & 44 & 1,22 & \multirow{2}{*}{0,936} \\
\hline & & Estudo & 27,20 & 26,55 & 4,96 & 24,30 & 29,05 & 20 & 2,17 & \\
\hline \multirow{12}{*}{$\begin{array}{l}\text { Relação S/R } \\
\text { dB NPS }\end{array}$} & \multirow{2}{*}{$1 \mathrm{kHz}$} & Controle & 6,82 & 5,05 & 7,56 & 0,00 & 9,28 & 44 & 2,23 & \multirow{2}{*}{0,277} \\
\hline & & Estudo & 3,87 & 3,15 & 3,81 & 0,25 & 5,70 & 20 & 1,67 & \\
\hline & \multirow{2}{*}{$1,5 \mathrm{kHz}$} & Controle & 16,09 & 15,65 & 7,97 & 12,05 & 20,90 & 44 & 2,36 & \multirow{2}{*}{0,268} \\
\hline & & Estudo & 14,08 & 12,90 & 9,18 & 5,28 & 18,43 & 20 & 4,02 & \\
\hline & \multirow{2}{*}{$2 \mathrm{kHz}$} & Controle & 19,03 & 19,65 & 6,06 & 14,48 & 23,80 & 44 & 1,79 & \multirow{2}{*}{0,885} \\
\hline & & Estudo & 18,89 & 17,90 & 8,19 & 13,43 & 23,95 & 20 & 3,59 & \\
\hline & \multirow{2}{*}{$3 \mathrm{kHz}$} & Controle & 19,81 & 20,20 & 5,76 & 15,98 & 23,10 & 44 & 1,70 & \multirow{2}{*}{0,215} \\
\hline & & Estudo & 22,23 & 22,20 & 8,20 & 17,00 & 28,48 & 20 & 3,59 & \\
\hline & \multirow{2}{*}{$4 \mathrm{kHz}$} & Controle & 16,29 & 15,75 & 6,16 & 11,85 & 19,80 & 44 & 1,82 & \multirow{2}{*}{$0,009^{*}$} \\
\hline & & Estudo & 21,66 & 22,55 & 7,81 & 16,03 & 28,13 & 20 & 3,42 & \\
\hline & \multirow{2}{*}{ Geral } & Controle & 14,60 & 14,55 & 5,24 & 10,33 & 17,78 & 44 & 1,55 & \multirow{2}{*}{0,913} \\
\hline & & Estudo & 15,00 & 13,75 & 7,20 & 9,80 & 20,73 & 20 & 3,16 & \\
\hline
\end{tabular}

Legenda: $\mathrm{EOAT}=$ emissões otoacústicas transientes; $\mathrm{kHz}=$ kilohertz; $\mathrm{S} / \mathrm{R}=$ sinal/ruído; $\mathrm{dB} \mathrm{NPS}=$ decibel nível de pressão sonora; $\mathrm{OD}=$ orelha direita; $\mathrm{OE}=$ orelha esquerda; $\mathbf{Q}$ = primeiro quartil; Q3 = terceiro quartil; $\mathrm{N}$ = número de sujeitos; IC = intervalo de confiança

* Valores estatisticamente significantes 
Tabela 4. Nível de resposta e relação S/R (em dB NPS) das EOAPD segundo o grupo por frequência

\begin{tabular}{|c|c|c|c|c|c|c|c|c|c|c|}
\hline EOAPD & & Grupo & Média & Mediana & Desvio padrão & Q1 & Q3 & $\mathrm{N}$ & IC & Valor de $p$ \\
\hline \multirow{4}{*}{$1,5 \mathrm{kHz}$} & \multirow{2}{*}{ nível } & Controle & 15,40 & 15,60 & 7,68 & 11,48 & 19,68 & 32 & 2,66 & \multirow{2}{*}{0,742} \\
\hline & & Estudo & 14,36 & 13,50 & 6,70 & 10,28 & 20,45 & 20 & 2,94 & \\
\hline & \multirow{2}{*}{$S / R$} & Controle & 8,84 & 7,00 & 5,88 & 6,18 & 12,03 & 32 & 2,04 & \multirow{2}{*}{0,910} \\
\hline & & Estudo & 7,90 & 7,05 & 4,95 & 6,38 & 9,75 & 20 & 2,17 & \\
\hline \multirow{4}{*}{$2 \mathrm{kHz}$} & \multirow{2}{*}{ nível } & Controle & 15,32 & 16,35 & 5,97 & 10,55 & 19,13 & 32 & 2,07 & \multirow{2}{*}{0,161} \\
\hline & & Estudo & 13,25 & 10,80 & 7,18 & 8,70 & 17,35 & 20 & 3,15 & \\
\hline & \multirow{2}{*}{$\mathrm{S} / \mathrm{R}$} & Controle & 11,90 & 10,20 & 6,20 & 7,08 & 17,00 & 32 & 2,15 & \multirow{2}{*}{0,553} \\
\hline & & Estudo & 10,91 & 9,15 & 7,91 & 6,88 & 14,35 & 20 & 3,46 & \\
\hline \multirow{4}{*}{$3 \mathrm{kHz}$} & \multirow{2}{*}{ nível } & Controle & 16,53 & 17,40 & 5,38 & 13,98 & 20,55 & 32 & 1,86 & \multirow{2}{*}{$0,027^{\star}$} \\
\hline & & Estudo & 12,09 & 13,40 & 7,37 & 6,45 & 17,53 & 20 & 3,23 & \\
\hline & \multirow{2}{*}{$S / R$} & Controle & 15,40 & 16,45 & 6,88 & 8,63 & 20,68 & 32 & 2,39 & \multirow{2}{*}{0,522} \\
\hline & & Estudo & 14,31 & 15,45 & 8,24 & 7,40 & 19,43 & 20 & 3,61 & \\
\hline \multirow{4}{*}{$4 \mathrm{kHz}$} & \multirow{2}{*}{ nível } & Controle & 16,55 & 18,00 & 7,31 & 10,93 & 22,10 & 32 & 2,53 & \multirow{2}{*}{0,435} \\
\hline & & Estudo & 14,36 & 18,25 & 8,98 & 11,53 & 20,65 & 20 & 3,94 & \\
\hline & \multirow{2}{*}{$S / R$} & Controle & 19,21 & 18,65 & 8,64 & 12,35 & 25,40 & 32 & 2,99 & \multirow{2}{*}{0,700} \\
\hline & & Estudo & 18,15 & 18,50 & 8,27 & 10,75 & 24,00 & 20 & 3,63 & \\
\hline \multirow{4}{*}{$6 \mathrm{kHz}$} & \multirow{2}{*}{ nível } & Controle & 16,99 & 19,75 & 7,62 & 9,73 & 21,50 & 32 & 2,64 & \multirow{2}{*}{$0,018^{\star}$} \\
\hline & & Estudo & 11,97 & 12,95 & 7,64 & 5,10 & 18,70 & 20 & 3,35 & \\
\hline & \multirow{2}{*}{$S / R$} & Controle & 18,85 & 18,75 & 8,29 & 11,48 & 26,03 & 32 & 2,87 & \multirow{2}{*}{0,247} \\
\hline & & Estudo & 16,03 & 17,60 & 8,15 & 12,05 & 20,48 & 20 & 3,57 & \\
\hline \multirow{4}{*}{8 kHz } & \multirow{2}{*}{ nível } & Controle & 24,39 & 28,85 & 9,91 & 19,35 & 31,08 & 32 & 3,43 & \multirow{2}{*}{$0,002^{*}$} \\
\hline & & Estudo & 16,62 & 17,75 & 9,01 & 9,18 & 23,98 & 20 & 3,95 & \\
\hline & \multirow{2}{*}{$S / R$} & Controle & 18,34 & 19,50 & 9,79 & 9,98 & 27,10 & 32 & 3,39 & \multirow{2}{*}{0,102} \\
\hline & & Estudo & 14,26 & 14,80 & 8,72 & 6,73 & 21,48 & 20 & 3,82 & \\
\hline
\end{tabular}

Legenda: EOAPD = emissões otoacústicas produto de distorção; kHz = kilohertz; $\mathrm{S} / \mathrm{R}=$ sinal/ruído; dB NPS = decibel nível de pressão sonora; OD = orelha direita; $\mathrm{OE}$ = orelha esquerda; $\mathrm{Q} 1$ = primeiro quartil; $\mathrm{Q} 3$ = terceiro quartil; $\mathrm{N}$ = número de sujeitos; IC = intervalo de confiança

* Valores estatisticamente significantes

\section{DISCUSSÃO}

Para compor a amostra do presente estudo, todos os lactentes expostos à medicação ototóxica $(\mathrm{N}=26)$ e todos lactentes sem qualquer indicador de risco auditivo $(\mathrm{N}=90)$, com nascimento na maternidade da ISCMSP, no período de fevereiro de 2007 a junho de 2007, foram convidados a participar da pesquisa. No entanto, a casuística deste estudo contou com apenas 26 lactentes para compor o grupo controle ( $28 \%$ de adesão) e 14 para compor o grupo estudo (53\% de adesão). O presente estudo apresenta tamanho de amostra semelhante ao de outros estudos realizados com população neonatal e uso de ototóxicos ${ }^{(8-9)}$.

Dificuldades na adesão à avaliação auditiva ambulatorial em dias pré-determinados também foram relatadas em outros estudos com lactentes ${ }^{(9-10)}$. Pode-se observar que, nos dias frios e chuvosos, a incidência do não comparecimento era maior que nos dias quentes e ensolarados; problemas sociais como falta de recursos financeiros para o transporte também constituíram um empecilho. Além disso, no grupo estudo, alguns lactentes encaminhados foram caracterizados como casos sociais: lactentes afastados temporariamente de suas famílias (institucionalizados), mães com drogadição e/ou moradoras de rua. No grupo comparação, assim como em outro estudo ${ }^{(9)}$, atingiu-se menor índice de adesão, apesar de as mães terem concordado com a realização do exame e participação na pesquisa, na alta hospitalar. Já no grupo estudo, as mães foram conscientizadas sobre a importância do exame, pelo longo tempo de internação e preocupação com a saúde geral do lactente.

Alguns estudos sobre os indicadores de risco auditivo apontam elevados índices de uso de medicação ototóxica no período neonatal ${ }^{(11)}$. Foi observado o uso de medicação ototóxica, durante a internação em unidade de terapia intensiva neonatal, como segundo indicador de risco para D.A., sendo que os medicamentos mais utilizados foram: vancomicina $(16,9 \%)$, aminoglicosídeos $(64,8 \%)$, furosemida $(14,2 \%)$, indometacina $(4,2 \%)$ e $21,1 \%$ receberam dois ou mais fármacos ototóxicos $\operatorname{associados}^{(12)}$. Outra pesquisa apontou a exposição ao uso de medicamentos ototóxicos como o indicador de risco predominante em seu grupo de estudo $(66,70 \%)$. No presente estudo, o uso de medicações ototóxicas teve a ocorrência $28,8 \%$ nos lactentes nascidos no período com indicadores de risco auditivo.

O presente estudo realizou a avaliação dos lactentes nascidos prematuros com idade gestacional corrigida entre $37 \mathrm{e}$ 41 semanas. Isto porque estudos prévios demonstraram que existe variação no padrão de resposta auditiva das EOA até o nascimento a termo, indicando maturação do sistema auditivo em recém-nascidos e a importância do uso da idade gestacional corrigida $^{(14-15)}$. 
$\mathrm{Na}$ literatura o uso das $\mathrm{EOAT}^{(10,12-20)}$ tem sido apontado como a melhor escolha para a triagem auditiva neonatal universal, já as EOAPD são priorizadas na monitoria da função auditiva $^{(21-22)}$.

No presente estudo foi utilizada a captação das EOA, tanto por transiente, quanto por produto de distorção. Sabe-se que o estímulo clique, usado na EOAT, avalia a cóclea somente até $4000 \mathrm{~Hz}$; no entanto, é o mais sensível para perdas leves. Já as EOAPD não são tão sensíveis para perdas de grau leve, mas avaliam as altas frequências. Assim sendo, optou-se por caracterizar os dois tipos de EOA na população estudada.

Diversos estudos ${ }^{(10,14,16,19,23)}$ apontaram maior nível das EOAT na fase neonatal, segundo gênero e orelha direita. No presente estudo, os resultados do grupo controle (Tabela 1) concordam com a literatura.

Já para o grupo estudo (Tabela 2), os níveis de resposta das EOAT da OE $(28,75 \mathrm{~dB})$ apresentaram resultados maiores do que a OD $(25,64 \mathrm{~dB})$ para o nível de resposta geral $(\mathrm{p}=0,009)$. Os resultados da relação $\mathrm{S} / \mathrm{R}$ das EOAT do grupo estudo também mostraram maiores valores para a orelha esquerda, porém sem significância estatística, estes resultados podem ser justificados pelo tamanho reduzido da amostra do grupo estudo.

$\mathrm{Na}$ análise das EOAPD de ambos os grupos não foram observadas diferenças estatisticamente significantes entre as orelhas e entre os gêneros. Isso concorda com os resultados obtidos por outros pesquisadores ${ }^{(6,8,21,24)}$.

Além disso, foi observado efeito de grupo no nível de resposta e na relação $\mathrm{S} / \mathrm{R}$ das EOAPD, confirmando a hipótese do presente estudo (Tabela 4). O grupo controle obteve maiores valores do que o grupo estudo com significância estatística nas frequências de 3000, 6000 e $8000 \mathrm{~Hz}$, sendo a maior significância em $8000 \mathrm{~Hz}(\mathrm{p}=0,002)$.

As diferenças observadas nas EOAPD entre os grupos coincidem com as altas frequências. Quando a lesão é cocleotóxica inicia-se no giro basal e com a continuidade da exposição progride para o ápice da cóclea ${ }^{(25)}$. A caracterização da alteração auditiva é observada em uma única ou em mais frequiências, na faixa de $2500 \mathrm{a} 8000 \mathrm{~Hz}$, sendo que o potencial de toxocidade do agente, dosagem, duração, método de exposição, suscetibilidade individual são fatores que influenciam na gravidade do caso.

No entanto, ainda existem controvérsias quanto ao efeito ototóxico dos aminoglicosídeos. Pesquisas experimentais recentes realizadas em cobaias ${ }^{(25)}$ verificaram que uma dose não ototóxica de amicacina, administrada previamente a uma dose ototóxica do mesmo antibiótico, tiveram efeito protetor. Estes resultados são promissores, sendo necessárias novas pesquisas em seres humanos que, por meio do uso controlado destes medicamentos, diminuiriam os riscos de provocar danos auditivos, verificados pelas EOA.

\section{CONCLUSÕES}

Nas EOAT apenas no grupo controle foram observados maiores níveis de resposta para a orelha direita e gênero feminino, porém sem significância estatística. Não foram observadas diferenças estatisticamente significantes entre os grupos controle e estudo

Nas EOAPD, tanto do grupo controle, quanto do grupo estudo, não foram observadas diferenças de gênero e orelha. Foram observados menores níveis de resposta e relação S/R para o grupo estudo, com valores estatisticamente significantes para as frequências de 3000, 6000 e $8000 \mathrm{~Hz}$ nas EOAPD.

A ação dos ototóxicos (gentamicina, amicacina e vancomicina) pode ser observada pela resposta típica das EOA em altas frequências, o que foi mais bem avaliado pelas EOAPD.

\section{AGRADECIMENTOS}

À Fundação de Amparo à Pesquisa do Estado de São Paulo (FAPESP), pelo apoio financeiro concedido por meio do projeto de iniciação científica $\mathrm{n}^{\circ}$ 0655139-0.

\begin{abstract}
Purpose: To analyze the otoacoustic emissions (OAE) of infants exposed to ototoxic drugs during the neonatal period. Methods: It was carried out the testing of transient OAE (TOAE) and distortion product OAE (DPOAE) with 40 infants: 14 infants exposed to ototoxic drugs for, at least, five days (study group), and 26 infants with no risks for hearing loss and no complications during the neonatal period (control group). The statistical analysis was performed using the non-parametric tests of Wilcoxon and Mann-Whitney, and the confidence interval for the average technique. Results: Lower levels of response and signal/noise relation of DPOAE were observed in the study group, with statistically significant values for the frequencies of 3000, 6000 and $8000 \mathrm{~Hz}$. Conclusion: The action of the ototoxic drugs can be observed by the typical response of OAE in high frequencies, which was better evaluated by the DPOAE.
\end{abstract}

Keywords: Infant; Risk factors; Otoacoustic emissions, spontaneous/drug effects

\title{
REFERÊNCIAS
}

1. Zocoli R, Reichow SL, Zocoli AM. Emissões otoacústicas x Cisplatina: detecção precoce da ototoxicidade em pacientes oncológicos. Rev Bras Otorrinolaringol. 2003;69(2):222-5.
2. Câmara MF. Efeito de fármacos ototóxicos na audição de recémnascidos de alto risco [tese]. São Paulo: Universidade de São Paulo, Faculdade de Medicina; 2005. 
3. Dishtchekenian A, Iorio MC, Petrilli AS, Azevedo MF. Monitorização auditiva na ototoxicidade. In: Barros AP, Arakawa L, Tonini MD, Carvalho VA, organizadores. Fonoaudiologia em cancerologia. São Paulo: Fundação Oncocentro de São Paulo; 2000. p.260-9.

4. Drogas ototóxicas [internet]. [cited 2009 may 1]. Disponível em: http:/ www.surdo.org.br.

5. Santos CF, Valete CM, Martins AG, Ferreira NG, Tomita S. Aspectos clínicos da ototoxicidade dos aminoglicosídios. Acta AWHO. 2000;19(3):160-4.

6. Vallejo JC, Silva MN, de Oliveira JA, Carneiro JJ, Rocha LS, Figueiredo JF, et al. Detecção precoce de ototoxicidade usando emissões otoacústicas produtivas de distorção. Rev Bras Otorrinolaringol. 2001;67(6):845-51.

7. American Academy of Pediatrics, Joint Committee on Infant Hearing. Year 2007 position statement: Principles and guidelines for early hearing detection and intervention programs. 2007;120(4):898-921.

8. Raineri GG, Coube CZ, Costa Filho OA, Alvarenga KF. Emissões otoacústicas evocadas - produto de distorção em neonatos audiologicamente normais. Rev Bras Otorrinolaringol. 2001;67(5):6448 .

9. Ruggieri-Marone M, Schochat E. Distortion product otoacoustic emissions in newborns treated by ototoxic drugs. Rev Laryngol Otol Rhinol (Bord). 2007;128(1-2):41-6.

10. Durante AS, Carvallo RM, Costa FS, Soares JC. Características das emissões otoacústicas por transientes em programa de triagem auditiva neonatal. Pro-Fono. 2005;17(2):133-40.

11. Korres S, Nikolopoulos TP, Komkotou V, Balatsouras D, Kandiloros D, Constantinou D, Ferekidis E. Newborn hearing screening: effectiveness, importance of high-risk factors, and characteristics of infants in the neonatal intensive care unit and well-baby nursery. Otol Neurotol. 2005;26(6):1186-90.

12. Sassada MM, Ceccon ME, Navarro JM, Vaz FA. Avaliação auditiva de recém-nascidos gravemente enfermos através do método de emissões otoacústicas evocadas transientes (EOAT) e audiometria de tronco cerebral (BERA). Pediatria (São Paulo). 2003;27(3):154-62.

13. Guerrero SM. Um estudo das respostas elétricas do tronco encefálico e das emissões otoacústicas evocadas transitórias em uma UTI neonatal [dissertação]. São Paulo: Pontifícia Universidade Católica de São Paulo; 2000.
14. Bassetto MC, Chiari BM, Azevedo MF. Emissões otoacústicas evocadas transientes (EOAET): amplitude da resposta em recém-nascidos a termo e pré-termo. Rev Bras Otorrinolaringol. 2003;69(1):84-92.

15. Chabert R, Guitton MJ, Amram D, Uziel A, Pujol R, Lallermant JG, Puel JL. Early maturation of evoked otoacoustic emissions and medial olivocochlear reflex in preterm neonates. Ped Res. 2006;59(2):305-8.

16. Newmark M, Merlob P, Bresloff I, Olsha M, Attias J. Click evoked otoacoustic emissions inter-aural and gender differences in newborns. J Basic Clin Physiol Pharmacol. 1997;8(3):134-9.

17. Paludetti G, Ottaviani F, Fetoni AR, Zuppa AA, Tortorolo G. Transient evoked otoacoustic emissions (TEOAEs) in newborns: normative data. Int J Ped Otorhinolaryngol. 1999;47(3):235-41.

18. Garcia CF, Isaac ML, Oliveira JA. Emissão otoacústica evocada transitória: instrumento para detecção precoce de alterações auditivas em recém-nascidos a termo e pré-termo. Rev Bras Otorrinolaringol. 2002;68(3):344-52.

19. Fuzetti CB, Lewis DR. Emissões otoacústicas espontâneas e evocadas por estímulo transiente em recém-nascidos. Pró-Fono. 2003;15(2):18998.

20. Jardim IS, Matas, CG, Carvalho RM. Emissões otoacústicas evocadas por estímulos transientes e potencial evocado auditivo de tronco encefálico automático na triagem auditiva neonatal. Einstein (São Paulo) 2008;6(3):253-61.

21. Lopes Filho O, Carlos R, Thomé D, Eckley C. Emissões otoacústicas transitórias e produtos de distorção na avaliação da audição em recém-nascidos com poucas horas de vida. Rev Bras Otorrinolaringol. 1996;62(3):220-8

22. Zang Z, Jiang ZD. Distortion product otoacoustic emissions during the first year in term infants: a longitudinal study. Brain Dev. 2007;29(6): 346-351.

23. Aidan D, Lestang P, Avan P, Bonfils P. Characteristics of transientevoked otoacoustic emissions (TEOES) in neonates. Acta Otolaryngol. 1997;117(1):25-30.

24. Pinto VS, Lewis DR. Emissões otoacústicas: produto de distorção em lactentes até dois meses de idade. Pró-Fono. 2007;19(2):195-204.

25. Oliveira JA, Canedo DM, Rossato M. Otoproteção das células ciliadas auditivas contra a ototoxicidade da amicacina. Rev Bras Otorrinolaringol. 2002;68(1):7-13. 